\title{
Characterization of Volatile Components of Eight FengHuang Dancong Manufactured Teas and Fresh Leaves by HS-SPME Coupled with GC-MS
}

\author{
Jingfang Shi ${ }^{1, \dagger}$, Wenjie Huang ${ }^{1, \dagger}$, Zhuang Chen ${ }^{1}$, Shili Sun ${ }^{2}$, Limin Xiang ${ }^{2}$, Qian Kong ${ }^{1}$, \\ Xiaohui Jiang ${ }^{2}$, Dong Chen ${ }^{2, *}$, Shijuan Yan ${ }^{1, *}$ \\ ${ }^{1}$ Agro-Biological Gene Research Center, Guangdong Academy of Agricultural Sciences, Guangzhou, China \\ ${ }^{2}$ Tea Research Institute, Guangdong Academy of Agricultural Sciences, Guangzhou, China
}

\section{Email address:}

shijingfang@agrogene.ac.cn (Jingfang Shi), huangwenjie@agrogene.ac.cn (Wenjie Huang), chenzhuang@agrogene.ac.cn (Zhuang Chen), sunshili@gdaas.cn (Shili Sun),767133613@qq.com (Xiaohui Jiang), kongqian@agrogene.ac.cn (Qian Kong), xianglimin88@163.com (Limin Xiang), chendong1113@sohu.com (Dong Chen), shijuan@agrogene.ac.cn (Shijuan Yan)

${ }^{*}$ Corresponding author

$\dagger$ Jingfang Shi and Wenjie Huang are co-first authors.

\section{To cite this article:}

Jingfang Shi, Wenjie Huang, Zhuang Chen, Shili Sun, Limin Xiang, Qian Kong, Xiaohui Jiang, Dong Chen, Shijuan Yan. Characterization of Volatile Components of Eight FengHuang Dancong Manufactured Teas and Fresh Leaves by HS-SPME Coupled with GC-MS. International Journal of Nutrition and Food Sciences. Vol. 7, No. 5, 2018, pp. 160-172. doi: 10.11648/j.jinfs.20180705.12

Received: August 15, 2018; Accepted: September 4, 2018; Published: October 9, 2018

\begin{abstract}
FengHuang Dancong tea is famous for its excellent aroma quality. In order to characterize the volatile components in different aroma types of FengHuang Dancong tea, both fresh leaves and manufactured teas of seven well-known aroma types and their ancestor variety, which were harvested from the same places and manufactured using the same procedure, were investigated using headspace solid-phase microextraction (HS-SPME) coupled with gas chromatography-mass spectrometry (GC-MS). Results indicated that the volatile composition and contents of manufactured teas and fresh leaves, including HuangZhi, XingRen, GuiHua, MiLan, JiangHua, YuLan YeLai and Fenghuang Shuixian, were obviously different. Linalool, (E)-2-hexenal, cis-3-hexenyl acetate, linalool oxide, methyl salicylate, geraniol, and nerolidol were the major volatile components in fresh leaves, and their total relative contents ranged from 78.44-90.07\%. But in manufactured teas, hotrienol, linalool, $\beta$-myrcene, D-limonene, 1-ethyl-1H-pyrrole-2-carboxaldehyde, $\beta$-ocimene, linalool oxide, benzyl nitrile, indole, jasmone, and nerolidol were the major volatile components, ranged from $60.12-93.97 \%$. Although there were some similarities in the aroma composition and content among the manufactured teas of different aroma types, each type had unique aroma characteristics. The obvious difference between FengHuang Shuixian and other aroma types of manufactured teas may be due to the higher content of alkene and pyrrole derivatives and lower content of alcohols, especial terpene alcohols. Furthermore, the correlations between manufactured teas and the fresh leaves indicated that the volatile compounds profile of fresh leaves may affect the aroma quality of the manufactured tea. This study provided a comprehensive comparison of the volatile profile in different aroma types of Fenghuang Dancong tea, which is a scientific foundation for further quality assessment of Fenghuang Dancong variety in the future.
\end{abstract}

Keywords: FengHuang Dancong, Volatile Components, HS-SPME, GC-MS

\section{Introduction}

Oolong tea is a semi-fermented Chinese traditional tea that dates back centuries and its unique characteristics are currently attracting more and more consumers worldwide [1]. Oolong tea combines the freshness of green tea with the fragrance of black tea. The numerous aromas of oolong tea are directly related to the type and extent of processing of the tea varieties [2]. Particular manufacturing processes give oolong tea unique flower and fruit flavors and variable aroma types [3].

In China, there are several famous oolong teas including Tie Guanyin tea, Wuyi rock tea, Dongding Oolong tea, and FengHuang Dancong [4]. FengHuang Dancong, one of the most famous oolong teas, is native to FengHuang town of Chao'an county in Guangdong Province [1]. FengHuang Dancong tea is famous for its compact appearance, high-quality aroma, and mellow taste. HuangZhi, XingRen, 
ZhiLan, GuiHua, MiLan, JiangHua, YuLan, YeLai, RouGui, and MoLi are the ten famous aroma types of FengHuang Dancong tea [5].

The quality of oolong tea can be evaluated using aroma, flavor, color, and appearance. Aroma and flavor are the two most important quality indices [6]. Many extraction techniques have been developed to analyze volatile components including direct solvent extraction, simultaneous distillation-extraction $\quad(\mathrm{SDE}) \quad[7, \quad 8], \quad$ steam distillation-liquid/liquid extraction [9], vacuum hydrodistillation [10], thermal desorption [10], and headspace solid-phase microextraction (HS-SPME) [7, 11, 12] SPME, a sensitive method that is economical and does not require solvents, integrates extraction and concentration in a single step [13]. SPME exhibits advantages in collecting actual volatile components, preventing the breakdown of heat-instable volatile components, and maintaining analytic stability Tea volatile components can be affected by tea variety, environmental factors, cultivation conditions, and processing conditions [14]. In the present study, the same environmental factors, cultivation conditions, and processing conditions were used to obtain different aroma types of FengHuang Dancong tea in order to determine varietal factors. Fresh leaves of eight types of Dancong tea, including MiLan, XingRen, YeLai, YuLan, JiangHua, GuiHua, HuangZhi, and FengHuang Shuixian, were collected and processed into eight types of FengHuang Dancong manufactured tea. Volatile components were analyzed with HS-SPME-GC-MS to investigate differences in aroma determinants between different types of FengHuang Dancong fresh leaves and manufactured teas. The differences in aroma composition and content between fresh leaves and manufactured teas were also investigated.

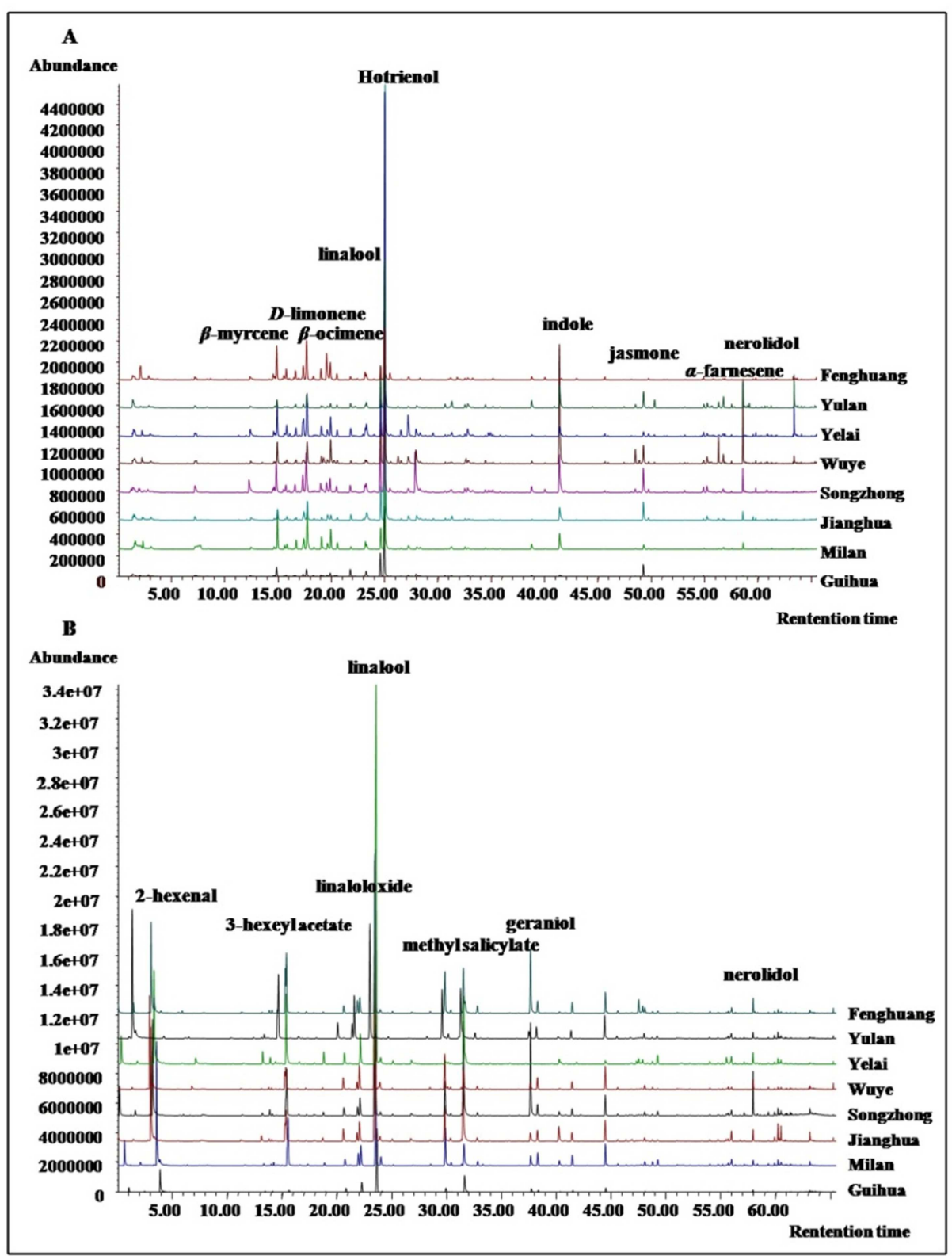

Figure 1. The total ion chromatograms of volatile components in FengHuang Dancong manufactured teas (A) and fresh leaves (B). 


\section{Results}

\subsection{Sensory Evaluation of FengHuang Dancong Manufactured Teas with Different Aroma Types}

Sensory evaluations were performed on extracted tea infusions from manufactured teas, including seven aroma types of FengHuang Dancong tea and the ancestral variety, FengHuang Shuixian. Results revealed that the major sensory characteristics for seven aroma types of FengHuang Dancong teas were flower-like aroma and honey-sweet taste, and FengHuang Shuixian had a sweet and pure aroma along with its fresh taste (Table 1). Compared with the seven aroma types of FengHuang Dancong tea, FengHuang Shuixian lacks the flower aroma and honey taste. All eight types of tea were long, thin and oily pieces, and the color of the brewed tea was light. All FengHuang Dancong manufactured teas in this study were evaluated as high quality teas (92.05-96.65 points) except FengHuang Shuixian (87.20 points).

\subsection{Comparison of Volatile Compounds among FengHuang Dancong Teas with Different Aroma Types}

The intensities of volatile compound peaks in the total ion chromatography (TIC) plot obtained from GC-MS analyses differed remarkably among the eight tea samples (Figure 1). A total of 82 peaks were identified based on the NIST mass spectral database, and the in-house database established using volatile authentic standards (Table 1 and Table A1).

Eighty-two volatile compounds in FengHuang Dancong oolong tea included aldehydes (6), alcohols (16), esters (8), ketones (5), alkenes (30), alkanes (5), and others (12) including indole and benzyl nitrile (Table 2). Of these compounds, the contents of alcohols (linalool or hotrienol) were significantly higher than the other ingredients both in fresh leaves and in manufactured teas except for FengHuang Shuixian. The major ingredients, however, were different in fresh leaves and manufactured teas (Figure 1).

Quantifications of volatile peaks were normalized based on signal intensity and used to perform multivariate statistical analyses. PCA with unsupervised pattern recognition was conducted to generate an overview of the metabolic pattern of eight groups of samples. As shown in Figure 2, there were significant differences in aromatic volatiles between the eight different types of Dancong teas both for fresh leaves and manufactured teas. Clear separation of samples was observed in MiLan, YeLai, YuLan, JiangHua, and HuangZhi fresh leaves, indicating that the volatile profiles were quite different among these five oolong varieties (Figure 2A). Consistent with the sensory evaluation results of Fenghuang Dancong manufactured teas, there were obvious differences in aromatic volatiles between FengHuang Shuixian and other tea types in the manufactured tea stage rather than in the fresh leaves stage (Figure 2B). The JiangHua type was clearly separated from the other aroma types (Figure 2B). Collectively, the results demonstrated that Fenghuang Dancong fresh leaves with different aroma types had different metabolic activity during oolong tea processing, although the same manufacturing process was used for all tea varieties.

Table 1. Sensory evaluation of the aroma of manufactured tea samples.

\begin{tabular}{|c|c|c|c|}
\hline & Shape $(15 \%)$ & Soup color $(10 \%)$ & Aroma $(30 \%)$ \\
\hline MiLan & Compact, long, thin strips yellow brown oily (95) & Bright oranger (95) & Honey, orchid aroma, long smell (94) \\
\hline XingRen & Compact, long, thin strips, light brown oily (92) & Bright oranger (94) & $\begin{array}{l}\text { Honeyand almond aroma, long and intense } \\
\text { smell (94) }\end{array}$ \\
\hline GuiHua & Compact, long, thin strips, yellow brown oily (95) & Clear, bright golden (96) & $\begin{array}{l}\text { Osmanthus and honey aroma, long and intense } \\
\text { smell (95.5) }\end{array}$ \\
\hline HuangZhi & Compact, long, thin strips, yellow brown oily (96) & Clear, bright golden (96) & $\begin{array}{l}\text { Gardenias and honey aroma, long and high } \\
\text { smell (97) }\end{array}$ \\
\hline YeLai & Compact, long, thin strips, light brown oily (92) & Bright golden (94) & Tuberose and honey aroma, long smell (90) \\
\hline YuLan & $\begin{array}{l}\text { Compact, long, thin strips, even, black brown oily } \\
\text { (90) }\end{array}$ & Light yellow (92) & $\begin{array}{l}\text { Magnolia randiflora and honey aroma, long and } \\
\text { pleasant smell (98) }\end{array}$ \\
\hline JiangHua & Tightly tired strip-type, yellow brown oily (95) & Bright golden (94) & Ginger and honey aroma, long smell (97) \\
\hline FengHuang Shuixian & Tightly tired strip-type (88) & Soft light orange (92) & Sweet and pure aroma, long smell (87) \\
\hline
\end{tabular}

Table 1. Continued.

\begin{tabular}{lll}
\hline & Taste (35\%) & Material (10\%) \\
\hline MiLan & Honey-sweet, full, fresh, infusion resistance (94) & Soft and bright, yellow red rimmed (95) \\
XingRen & Mellow, full, mi yun, infusion resistance (95) & Soft, red rimmed (92) \\
GuiHua & Honey-sweet, full, fresh, infusion resistance (96) & Soft, even, yellowish green, red rimmed (95) \\
HuangZhi & Honey-sweet, delicate, full, fresh, infusion resistance (97) & Soft, even, light yellow, red rimmed (96) \\
YeLai & Mellow, fresh, shanyun, infusion resistance (93) & Soft and bright, brown red rimmed (93) \\
YuLan & Mellow, fresh, shanyun, infusion resistance (93) & Soft and bright, yellow red rimmed (92) \\
JiangHua & Mellow, full, fresh and sweet, infusion resistance (95) & Soft and bright, yellow red rimmed (94) \\
FengHuang Shuixian & Taste great, fresh (86) & Soft and bright, red rimmed (86) \\
\hline
\end{tabular}


A

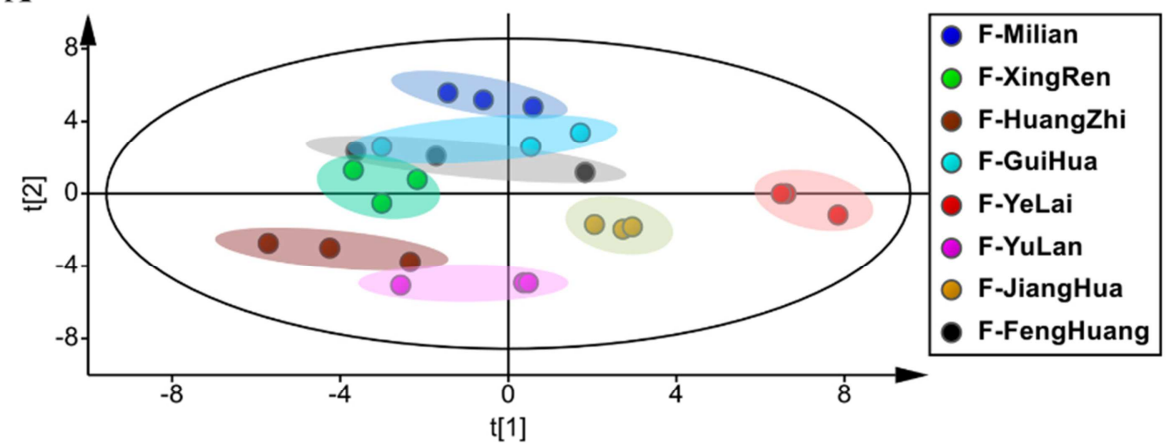

B

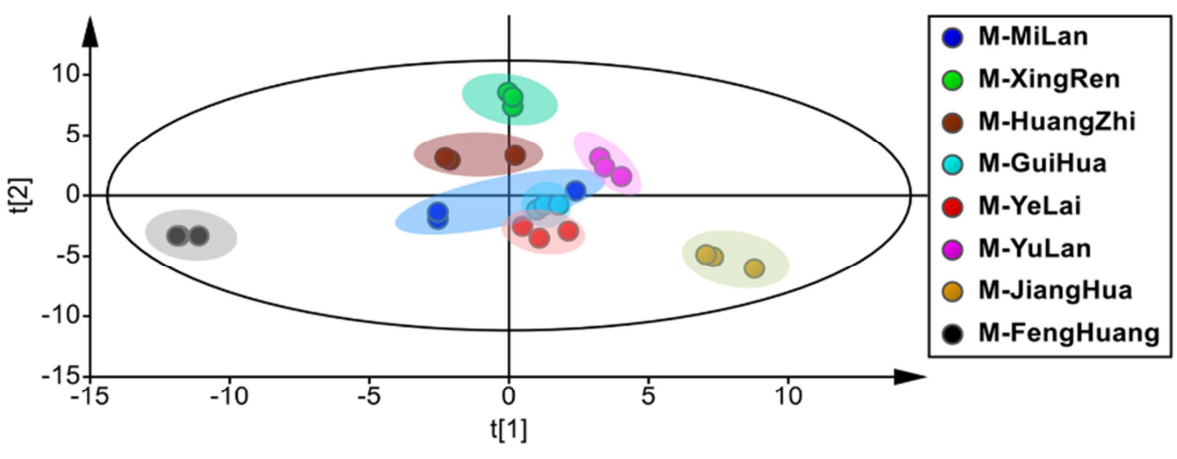

Figure 2. Volatile compound profiles of fresh leaves and manufactured teas of eight FengHuang Dancong varieties.

\subsection{Comparison of Volatile Compounds Among Fresh Leaves and Manufactured Teas with Different Aroma Types}

As shown in Figure 3, qualitative and quantitative differences were observed in volatile components between manufactured teas and fresh leaves. ANOVA was used to distinguish different tea samples by their aroma composition. Alcohols were the most important class of volatile components in both fresh leaves and manufactured teas except in FengHuang Shuixian manufactured tea. Aldehydes and esters were identified as the second most important flavoring components of fresh leaves while alkenes and other compounds such as indole, benzyl nitrile, and pyrrole derivatives were important for flavor of the manufactured teas.

Among aroma-active compounds, linalool, (E)-2-hexenal, cis-3-hexenyl acetate, linalool oxide, methyl salicylate, geraniol, and nerolidol were the major volatile components in fresh leaves, demonstrating relative percentages as high as 78.44-90.07\% (Table 2). Hotrienol, linalool, $\beta$-myrcene, D-limonene, 1-ethyl-1H-pyrrole-2-carboxaldehyde, $\beta$-ocimene, linalool oxide, benzyl nitrile, indole, jasmone, and nerolidol were the major volatile components in manufactured teas and their total relative content ranged from 60.12-93.97\% (Table 2). Although each volatile component changed in dependently after processing, three types of changing patterns were observed.
First, some components were only detectable, or increased, in the manufactured teas, such as hotrienol, 1-ethyl-1H-pyrrole and 1-ethyl-1H-pyrrole-2-carboxaldehyde. The content of hotrienol (28.30-41.20\%) was highest in manufactured tea (Table 2). However, in fresh leaves, hotrienol was not detected and but linalool was at the highest concentration (34.20-42.00\%) (Table 2). 1-ethyl-1H-pyrrole and 1-ethyl-1 $H$-pyrrole-2-carboxaldehyde were not detected in fresh leaves but were detected in manufactured tea; content of 1-ethyl- $1 \mathrm{H}$-pyrrole was $0.55-5.82 \%$ and content of 1-ethyl- $1 H$-pyrrole-2-carboxaldehyde was $0.53-9.20 \%$ (Table 2). Indole (0-19.54\%) was another volatile compound found at relatively high concentration in manufactured teas but not detected in fresh leaves. Phenylpropanoid/benzenoid derivatives such as phenylethyl alcohol (0-0.93\%), benzaldehyde (0.65-3.09\%), benzene acetaldehyde (0-0.83\%), and benzyl nitrile (0-9.64\%) also increased after the processing procedure (Table 2); they are important contributors to the fruity and floral smells in manufactured teas [15].

Second, there were some components that decreased in the manufactured teas, such as methyl salicylate (0-1.78\%), hexenal $(0 \%)$, hexenol $(0 \%)$, and cis-3-hexenyl acetate $(0 \%)$, which were the major component in fresh leaves. Alcohols exhibited the third changing pattern (Table 2). The relative abundance of mainly linalool, nerolidol, and geraniol varied during processing. However, the total content of alcoholonly decreased largely in FengHuang Shuixian. 
Table 2. Volatile compounds and their relative contents in eight aroma types FengHuang Dancong fresh leaves and manufactured teas.

\begin{tabular}{|c|c|c|c|c|c|c|c|c|c|c|}
\hline & \multirow{4}{*}{$\begin{array}{l}\text { Retention } \\
\text { time (min) }\end{array}$} & \multirow[b]{3}{*}{ Volatile } & \multicolumn{8}{|c|}{ Relative percentage content [\%] } \\
\hline & & & \multicolumn{8}{|c|}{ Fresh leaves } \\
\hline & & & Mi & Xing & Gui & Huang & Ye & Yu & Jiang & Feng \\
\hline & & components & Lan & Ren & Hua & Zhi & Llai & Lan & Hua & Huang \\
\hline Alcohol & & & & & & & & & & \\
\hline 1 & 3.88 & 3-Hexen-1-ol & 0.43 & 1.11 & 0.86 & 1.5 & 1.02 & 2.85 & 0 & 3.55 \\
\hline 2 & 7.39 & 2-Heptanol & 0 & 0 & 0 & 0 & 0.66 & 0 & 0.78 & 0 \\
\hline 3 & 13.35 & 1-Octen-3-ol & 0.4 & 1.09 & 0.55 & 0.54 & 1.03 & 1.19 & 0 & 0.22 \\
\hline 4 & 18.37 & 2-Ethyl-1-hexanol & 0 & 0 & 0 & 0.47 & 0 & 0.36 & 0 & 0 \\
\hline 5 & 21.88 & Linaloloxide I & 1.13 & 2.41 & 2.19 & 1.6 & 0.79 & 0.9 & 3.06 & 1.62 \\
\hline 6 & 23.3 & Linaloloxide II & 2.43 & 3.93 & 5.33 & 4.94 & 3.11 & 1.98 & 5.96 & 3.45 \\
\hline 7 & 24.68 & Linalool & 41.92 & 35.92 & 42.02 & 34.16 & 53.71 & 41.56 & 50.95 & 36.38 \\
\hline 8 & 25.04 & Hotrienol & 0 & 0 & 0 & 0 & 0 & 0 & 0 & 0 \\
\hline 9 & 25.51 & Phenylethyl alcohol & 0 & 0 & 0 & 0 & 0 & 0 & 0 & 0 \\
\hline 10 & 29.96 & Pyranoid linalool oxide & 0 & 0 & 0 & 0 & 0.36 & 0 & 0 & 0 \\
\hline 11 & 32.6 & $\alpha$-Terpineol & 0 & 0 & 0 & 0 & 0.12 & 0 & 0 & 0 \\
\hline 12 & 38.81 & Geraniol & 12.56 & 1.87 & 2.73 & 3.57 & 0.17 & 1.42 & 1.48 & 10.95 \\
\hline 13 & 58.62 & Nerolidol & 3.21 & 1.19 & 1.04 & 1.08 & 1.01 & 1.02 & 1.24 & 1.52 \\
\hline 14 & 60.24 & $\alpha$-cadinol & 0.56 & 1.68 & 0.5 & 0.86 & 0.4 & 0 & 0.27 & 0.42 \\
\hline 15 & 60.5 & tau-Muurolol & 0.24 & 1.17 & 0 & 0.59 & 0.23 & 0 & 0.19 & 0 \\
\hline 16 & 65.25 & Phytol & 0.04 & 0.13 & 0.13 & 0 & 0.11 & 0.15 & 0.29 & 0.21 \\
\hline Total & & & 62.91 & 50.51 & 55.35 & 49.31 & 62.71 & 51.43 & 64.22 & 58.3 \\
\hline Alkene & & & & & & & & & & \\
\hline 1 & 14.46 & cis-2,6-Dimethyl-2,6-octadiene & 0 & 0 & 0 & 0 & 0 & 0 & 0 & 0 \\
\hline 2 & 14.99 & $\beta$-Myrcene & 0.57 & 0.35 & 0.36 & 0.64 & 0.51 & 0.33 & 0.56 & 0.54 \\
\hline 3 & 15.62 & $\alpha$-Phellandrene & 0 & 0 & 0 & 0 & 0.35 & 0 & 0 & 0 \\
\hline 4 & 15.87 & 2,3,6-Trimethyl-1,5-heptadiene & 0 & 0 & 0 & 0 & 0 & 0 & 0 & 0 \\
\hline 5 & 16.73 & $\alpha$-Terpinene & 0 & 0 & 0 & 0 & 0 & 0 & 0 & 0 \\
\hline 6 & 17.47 & $o$-Cymene & 0 & 0 & 0 & 0 & 0 & 0 & 0 & 0 \\
\hline 7 & 17.79 & D-Limonene & 0.2 & 0.14 & 0.15 & 0.22 & 0.23 & 0.18 & 0.28 & 0.29 \\
\hline 8 & 19.13 & trans- $\beta$-Ocimene & 0.15 & 0 & 0.13 & 0 & 0.12 & 0 & 0.15 & 0.18 \\
\hline 9 & 20.01 & $\beta$-Ocimene & 0.46 & 0.57 & 0.36 & 0.7 & 1.27 & 0.28 & 0.36 & 0.32 \\
\hline 10 & 20.59 & $\gamma$-Terpinene & 0 & 0 & 0 & 0 & 0 & 0 & 0 & 0 \\
\hline 11 & 23.23 & Terpinolene & 0 & 0 & 0 & 0 & 0 & 0 & 0 & 0 \\
\hline 12 & 26.54 & $1,3,8-p$-Menthatriene & 0 & 0 & 0 & 0 & 0 & 0 & 0 & 0 \\
\hline 13 & 27.29 & Cosmene & 0 & 0 & 0 & 0 & 0 & 0 & 0 & 0 \\
\hline 14 & 28.33 & Neo-allo-ocimene & 0 & 0 & 0 & 0 & 0 & 0 & 0 & 0 \\
\hline 15 & 39.65 & 1-Methyl-naphthalene, & 0 & 0 & 0 & 0 & 0.05 & 0.08 & 0 & 0 \\
\hline 16 & 44.66 & $\alpha$-Cubebene & 0.1 & 0.16 & 0 & 0.38 & 0.09 & 0 & 0 & 0.25 \\
\hline 17 & 45.64 & Longicyclene & 0.22 & 0.3 & 0.2 & 0.5 & 0 & 0.41 & 0.42 & 0.32 \\
\hline 18 & 45.66 & 1, 1, 5-Trimethyl-1, 2-dihydronaphthalene & 0 & 0 & 0 & 0 & 0 & 0 & 0 & 0 \\
\hline 19 & 45.95 & Ionene & 0 & 0 & 0 & 0 & 0 & 0 & 0 & 0 \\
\hline 20 & 47.44 & Copaene & 0 & 0.25 & 0.12 & 0.27 & 0 & 0 & 0 & 0 \\
\hline 21 & 48.1 & Longifolene & 0.46 & 0.6 & 0.53 & 0.95 & 0 & 0.78 & 0.8 & 0.72 \\
\hline 22 & 50.31 & Caryophyllene & 0.6 & 0.34 & 0 & 1.49 & 1.02 & 0 & 0 & 0.5 \\
\hline 23 & 54.77 & $\alpha$-Muurolene & 0.11 & 0.19 & 0.12 & 0.2 & 0.07 & 0 & 0.13 & 0.15 \\
\hline 24 & 55.94 & trans-Calamenene & 0 & 0.15 & 0 & 0 & 0 & 0 & 0 & 0 \\
\hline 25 & 56.32 & $\alpha$-Farnesene & 0 & 0 & 0 & 0.45 & 1.14 & 0 & 0 & 0.4 \\
\hline 26 & 56.01 & $\delta$-Cadinene & 0.65 & 1.5 & 0.44 & 1.32 & 0.85 & 0.24 & 0.43 & 0.95 \\
\hline 27 & 56.79 & $\alpha$-Calacorene & 0.08 & 0.18 & 0 & 0 & 0.08 & 0 & 0 & 0.09 \\
\hline 28 & 59.9 & Cubenene & 0.29 & 0 & 0 & 0.34 & 0.16 & 0 & 0.09 & 0 \\
\hline 29 & 59.98 & Alloaromadendrene & 0.08 & 0 & 0 & 0 & 0 & 0 & 0 & 0 \\
\hline 30 & 60.88 & Cadalene & 0.16 & 0.09 & 0.09 & 0.09 & 0.03 & 0.19 & 0.14 & 0.1 \\
\hline Total & & & 4.14 & 4.83 & 2.49 & 7.54 & 5.97 & 2.49 & 3.36 & 4.82 \\
\hline Ketone & & & & & & & & & & \\
\hline 1 & 14.62 & 6-Methyl-5-hepten-2-one & 0 & 0 & 0 & 0 & 0 & 0 & 0 & 0 \\
\hline 2 & 26.26 & 2-Methyl-6-methylene-1,7-Octadie-3-one & 0 & 0.38 & 0 & 0 & 0.4 & 0.15 & 0 & 0 \\
\hline 3 & 49.31 & Jasmone & 0.18 & 0 & 0 & 0 & 0.19 & 0 & 0 & 0 \\
\hline 4 & 54.93 & trans- $\beta$-Ionone & 0.17 & 0.28 & 0.25 & 0.37 & 0.36 & 0.39 & 0.36 & 0.28 \\
\hline 5 & 55.25 & Jasmine lactone & 0 & 0 & 0 & 0 & 0 & 0 & 0 & 0 \\
\hline Total & & & 0.34 & 0.66 & 0.25 & 0.37 & 0.95 & 0.54 & 0.36 & 0.28 \\
\hline Ester & & & & & & & & & & \\
\hline 1 & 15.47 & 3-Hexen-1-ol, acetate & 1.84 & 2.76 & 2.51 & 2.88 & 9.7 & 7.87 & 1.94 & 7.34 \\
\hline 2 & 22.65 & Benzoic acid, methyl ester & 0 & 0 & 0 & 0 & 0 & 0.3 & 0 & 0 \\
\hline 3 & 28.58 & Acetic acid, 2-ethylhexyl ester & 0.22 & 0.3 & 0.26 & 0.54 & 0 & 0.43 & 0.38 & 0.25 \\
\hline 4 & 32.85 & Methyl salicylate & 14.87 & 14.62 & 20.14 & 6.43 & 7.18 & 4.65 & 6.25 & 8.47 \\
\hline 5 & 48.5 & Hexanoic acid, 3-hexenyl ester, (Z)- & 0 & 0 & 0 & 0 & 0.59 & 0 & 0 & 2.24 \\
\hline 6 & 48.89 & Hexanoic acid, hexyl ester & & & & & & & & \\
\hline 7 & 63.35 & Phthalic acid, diisobutyl ester & 0 & 0 & 0 & 0 & 0.1 & 0 & 0.06 & 0.13 \\
\hline 8 & 64.07 & Hexadecanoic acid, methyl ester & & & & & & & & \\
\hline Total & & & 16.94 & 17.68 & 22.91 & 9.85 & 17.57 & 13.25 & 8.63 & 18.43 \\
\hline Alhyde & & & & & & & & & & \\
\hline 1 & 3.58 & 2-Hexenal & 11.42 & 18.72 & 14.1 & 23.77 & 9.26 & 24.34 & 17.33 & 12.9 \\
\hline 2 & 12.49 & Benzaldehyde & 0.29 & 0.47 & 0.28 & 0.35 & 0.24 & 0.27 & 0.43 & 0.54 \\
\hline 3 & 19.28 & Benzeneacetaldehyde & 0.16 & 0.25 & 0 & 0 & 0 & 0 & 0 & 0 \\
\hline 4 & 24.04 & Nonanal & 0.54 & 0.92 & 1.16 & 1.91 & 0.92 & 1.26 & 1.66 & 0.74 \\
\hline 5 & 34.43 & Decanal & 0 & 0 & 0.19 & 0.27 & 0.15 & 0.3 & 0.3 & 0 \\
\hline 6 & 34.73 & 1-p-Menthen-9-al & 0 & 0 & 0 & 0 & 0.06 & 0 & 0 & 0 \\
\hline Total & & & 12.41 & 20.36 & 15.74 & 26.31 & 10.63 & 26.17 & 19.71 & 14.18 \\
\hline Other & & & & & & & & & & \\
\hline
\end{tabular}




\begin{tabular}{|c|c|c|c|c|c|c|c|c|c|c|}
\hline & & & \multicolumn{8}{|c|}{ Relative percentage content [\%] } \\
\hline & & & \multicolumn{8}{|c|}{ Fresh leaves } \\
\hline 1 & 2.32 & 1-ethyl-1 $H$-Pyrrole & 0 & 0 & 0 & 0 & 0 & 0 & 0 & 0 \\
\hline 2 & 3.98 & $p$-Xylene & 0 & 0 & 0 & 0 & 0 & 0.29 & 0 & 0.41 \\
\hline 3 & 19.68 & 1-ethyl-1 $H$-Pyrrole-2-carboxaldehyde & 0 & 0 & 0 & 0 & 0 & 0 & 0 & 0 \\
\hline 4 & 23.31 & 1-Methyl-4-(1-methylethenyl)-benzene & 0 & 0 & 0 & 0 & 0 & 0 & 0 & 0 \\
\hline 5 & 25.53 & 4-Methoxy-N-methyl-benzenamine & 0 & 0 & 0 & 0 & 0 & 0 & 0 & 0 \\
\hline 6 & 28.06 & Benzyl nitrile & 0.38 & 0.73 & 0 & 0.15 & 0.82 & 0 & 0 & 0.27 \\
\hline 7 & 29.55 & Nerol oxide & 0 & 0 & 0 & 0 & 0 & 0 & 0 & 0 \\
\hline 8 & 41.42 & Indole & 0.53 & 2.85 & 0.62 & 1.13 & 0.69 & 1.4 & 0.22 & 0.34 \\
\hline 9 & 55.8 & Dibenzofuran & 0 & 0 & 0 & 0 & 0 & 0 & 0 & 0 \\
\hline 10 & 58.36 & Caryophyllene oxide & 0.26 & 0 & 0 & 0.36 & 0 & 0 & 0 & 0 \\
\hline 11 & 60.64 & 2,2',5,5'-Tetramethyl-1,1'-biphenyl & 0.04 & 0 & 0 & 0 & 0 & 0.1 & 0 & 0 \\
\hline 12 & 63.12 & Caffeine & 0.39 & 0.6 & 0.3 & 0.44 & 0.27 & 0.54 & 0.32 & 0.51 \\
\hline total & & & 1.61 & 4.18 & 0.92 & 2.09 & 1.77 & 2.34 & 0.54 & 1.53 \\
\hline \multicolumn{11}{|c|}{ Alkane } \\
\hline 1 & 32.87 & Dodecane & 0.38 & 0.49 & 0.45 & 1.04 & 0 & 0.74 & 0.72 & 0.68 \\
\hline 2 & 41.49 & Tridecane & 0.77 & 1.07 & 1.1 & 1.98 & 0 & 1.44 & 1.57 & 1.37 \\
\hline 3 & 49.76 & Tetradecane & 0.22 & 0.21 & 0.49 & 0.87 & 0.3 & 0.49 & 0.44 & 0.27 \\
\hline 4 & 55.19 & Pentadecane & 0 & 0 & 0 & 0.28 & 0 & 0.34 & 0.17 & 0 \\
\hline 5 & 59.36 & Hexadecane & 0.28 & 0 & 0.3 & 0.36 & 0.09 & 0.77 & 0.27 & 0.15 \\
\hline Total & & & 1.65 & 1.77 & 2.34 & 4.53 & 0.39 & 3.78 & 3.18 & 2.47 \\
\hline
\end{tabular}

\begin{tabular}{|c|c|c|c|c|c|c|c|c|c|c|}
\hline & \multicolumn{10}{|c|}{ Relative percentage content [\%] } \\
\hline & \multirow{3}{*}{$\begin{array}{l}\text { Retention } \\
\text { time (min) }\end{array}$} & \multirow[b]{2}{*}{ Volatile } & \multicolumn{8}{|c|}{ Manufactured tea } \\
\hline & & & Mi & Xing & Gui & Huang & Ye & $\mathbf{Y u}$ & Jiang & Feng \\
\hline & & components & Lan & Ren & Hua & Zhi & Llai & Lan & Hua & Huang \\
\hline \multicolumn{11}{|l|}{ Alcohol } \\
\hline 1 & 3.88 & 3-Hexen-1-ol & 0 & 0 & 0 & 0 & 0 & 0 & 0 & 0 \\
\hline 2 & 7.39 & 2-Heptanol & 0 & 0 & 0 & 0 & 0 & 0 & 0 & 0 \\
\hline 3 & 13.35 & 1-Octen-3-ol & 0 & 0 & 0 & 0 & 0 & 0 & 0 & 0 \\
\hline 4 & 18.37 & 2-Ethyl-1-hexanol & 0 & 0 & 0.69 & 0 & 0 & 0 & 0.16 & 0.94 \\
\hline 5 & 21.88 & Linaloloxide I & 0.76 & 0.45 & 4.67 & 1.57 & 1.25 & 1.4 & 1.22 & 1.03 \\
\hline 6 & 23.3 & Linaloloxide II & 0 & 0 & 4.51 & 0 & 0 & 1.95 & 2.04 & 1.63 \\
\hline 7 & 24.68 & Linalool & 5.31 & 9.09 & 12.78 & 8.08 & 6.93 & 11.3 & 5.46 & 3.66 \\
\hline 8 & 25.04 & Hotrienol & 40.72 & 25.26 & 38.91 & 28.33 & 48.35 & 41.18 & 72.56 & 15.66 \\
\hline 9 & 25.51 & Phenylethyl alcohol & 0 & 0.34 & 0 & 0.93 & 0 & 0 & 0 & 0 \\
\hline 10 & 29.96 & Pyranoid linalool oxide & 0.62 & 0 & 0 & 0 & 0.81 & 2.53 & 0 & 0 \\
\hline 11 & 32.6 & $\alpha$-Terpineol & 0.76 & 0 & 0 & 0.77 & 0.46 & 0.91 & 0.28 & 0.43 \\
\hline 12 & 38.81 & Geraniol & 1.75 & 0.41 & 0.87 & 0.93 & 0.15 & 1.94 & 0 & 0.47 \\
\hline 13 & 58.62 & Nerolidol & 1.2 & 6.73 & 0.37 & 1.94 & 0.13 & 3.49 & 0.73 & 0 \\
\hline 14 & 60.24 & $\alpha$-cadinol & 0 & 0 & 0 & 0 & 0 & 0 & 0 & 0 \\
\hline 15 & 60.5 & tau-Muurolol & 0 & 0 & 0 & 0 & 0 & 0 & 0 & 0 \\
\hline 16 & 65.25 & Phytol & 0 & 0 & 0 & 0 & 0 & 0 & 0 & 0 \\
\hline & \multicolumn{10}{|c|}{ Alkene } \\
\hline 1 & 14.46 & cis-2,6-Dimethyl-2,6-octadiene & 0 & 0.24 & 0.69 & 0.41 & 0.59 & 0 & 0 & 1.78 \\
\hline 2 & 14.99 & $\beta$-Myrcene & 5.96 & 2.99 & 5.22 & 3.84 & 2.88 & 1.89 & 1.42 & 9.86 \\
\hline 3 & 15.62 & $\alpha$-Phellandrene & 0.61 & 0.25 & 0.42 & 0.3 & 1.48 & 0.27 & 0.2 & 1.41 \\
\hline 4 & 15.87 & 2,3,6-Trimethyl-1,5-heptadiene & 0.96 & 0.66 & 1.12 & 0.94 & 1.16 & 0.05 & 0.48 & 3.27 \\
\hline 5 & 16.73 & $\alpha$-Terpinene & 1.4 & 0.71 & 0.73 & 1 & 1.07 & 0.93 & 0.35 & 2.71 \\
\hline 6 & 17.47 & $o$-Cymene & 3.05 & 1.08 & 1.51 & 2.83 & 2.8 & 0.79 & 1.42 & 4.72 \\
\hline 7 & 17.79 & D-Limonene & 8.21 & 3.71 & 5.2 & 6.31 & 6.67 & 3.8 & 3.07 & 13.93 \\
\hline 8 & 19.13 & trans- $\beta$-Ocimene & 2.01 & 0.89 & 1.17 & 0.86 & 1.06 & 0.5 & 0.32 & 3 \\
\hline 9 & 20.01 & $\beta$-Ocimene & 3.84 & 2.9 & 2.48 & 1.72 & 2.28 & 1.12 & 0.52 & 5.14 \\
\hline 10 & 20.59 & $\gamma$-Terpinene & 1 & 0.49 & 0.83 & 0.59 & 0.81 & 0.74 & 0.33 & 1.93 \\
\hline 11 & 23.23 & Terpinolene & 1.21 & 0.75 & 0 & 0.7 & 0.96 & 0.63 & 0 & 2.26 \\
\hline 12 & 26.54 & $1,3,8$ - $p$-Menthatriene & 0 & 0.41 & 0 & 0 & 0.75 & 0 & 0 & 0 \\
\hline 13 & 27.29 & Cosmene & 1.51 & 1.31 & 1.11 & 0.83 & 3.19 & 0 & 0.71 & 1.38 \\
\hline 14 & 28.33 & Neo-allo-ocimene & 0 & 0.59 & 0.6 & 1.13 & 0.71 & 0 & 0 & 0 \\
\hline 15 & 39.65 & 1-Methyl-naphthalene, & 0 & 0 & 0 & 0 & 0 & 0 & 0 & 0 \\
\hline 16 & 44.66 & $\alpha$-Cubebene & 0 & 0 & 0 & 0 & 0 & 0 & 0 & 0 \\
\hline 17 & 45.64 & Longicyclene & 0 & 0 & 0 & 0 & 0 & 0 & 0 & 0 \\
\hline 18 & 45.66 & 1, 1, 5-Trimethyl-1, 2-dihydronaphthalene & 0 & 0.33 & 0 & 0.27 & 0.37 & 0 & 0 & 0.58 \\
\hline 19 & 45.95 & Ionene & 0 & 0.18 & 0 & 0.1 & 0 & 0 & 0 & 0 \\
\hline 20 & 47.44 & Copaene & 0 & 0.19 & 0 & 0 & 0 & 0.28 & 0 & 0 \\
\hline 21 & 48.1 & Longifolene & 0 & 0 & 0 & 0 & 0 & 0 & 0 & 0 \\
\hline 22 & 50.31 & Caryophyllene & 0.21 & 0.16 & 0 & 0 & 0.22 & 1.8 & 0 & 0 \\
\hline 23 & 54.77 & $\alpha$-Muurolene & 0 & 0 & 0 & 0 & 0 & 0 & 0 & 0 \\
\hline 24 & 55.94 & trans-Calamenene & 0 & 0 & 0 & 0 & 0 & 0 & 0 & 0 \\
\hline 25 & 56.32 & $\alpha$-Farnesene & 0 & 1.8 & 0.25 & 0 & 0.15 & 1.11 & 0.17 & 0 \\
\hline 26 & 56.01 & $\delta$-Cadinene & 0.35 & 1.32 & 0 & 0.48 & 0 & 2.37 & 0 & 0 \\
\hline 27 & 56.79 & $\alpha$-Calacorene & 0.25 & 0.23 & 0 & 0 & 0 & 0.5 & 0 & 0 \\
\hline 28 & 59.9 & Cubenene & 0 & 0 & 0 & 0 & 0 & 0 & 0 & 0 \\
\hline 29 & 59.98 & Alloaromadendrene & 0 & 0 & 0 & 0 & 0 & 0 & 0 & 0 \\
\hline 30 & 60.88 & Cadalene & 0.1 & 0.08 & & 0.06 & 0.1 & 0 & 0 & 0 \\
\hline Total & & & 30.66 & 21.29 & 21.32 & 22.38 & 27.25 & 16.78 & 8.99 & 51.97 \\
\hline \multicolumn{11}{|l|}{ Ketone } \\
\hline 1 & 14.62 & 6-Methyl-5-hepten-2-one & 0 & 0.26 & 0.62 & 0.64 & 0.36 & 0.44 & 0 & 0.83 \\
\hline 2 & 26.26 & 2-Methyl-6-methylene-1,7-Octadie-3-one & 0 & 1.01 & 0 & 0 & 0 & 0.37 & 0 & 0 \\
\hline 3 & 49.31 & Jasmone & 1.72 & 3.1 & 6.63 & 3.83 & 0.79 & 3.88 & 2.43 & 0 \\
\hline
\end{tabular}




\begin{tabular}{|c|c|c|c|c|c|c|c|c|c|c|}
\hline & & & \multicolumn{8}{|c|}{ Relative percentage content [\%] } \\
\hline & & & \multicolumn{8}{|c|}{ Manufactured tea } \\
\hline 4 & 54.93 & trans- $\beta$-Ionone & 0 & 0.51 & 0.46 & 0.63 & 0.52 & 0.67 & 0.15 & 0.34 \\
\hline 5 & 55.25 & Jasmine lactone & 0 & 1.08 & 0 & 0.9 & 0.43 & 0 & 0 & 0 \\
\hline $\begin{array}{l}\text { Total } \\
\text { Ester }\end{array}$ & & & 1.72 & 5.97 & 7.71 & 6 & 2.1 & 5.35 & 2.58 & 1.17 \\
\hline 1 & 15.47 & 3-Hexen-1-ol, acetate & 0 & 0 & 0 & 0 & 0 & 0 & 0 & 0 \\
\hline 2 & 22.65 & Benzoic acid, methyl ester & 0 & 0 & 0 & 0 & 0 & 0 & 0 & 0 \\
\hline 3 & 28.58 & Acetic acid, 2-ethylhexyl ester & 0 & 0 & 0 & 0 & 0 & 0 & 0 & 0 \\
\hline 4 & 32.85 & Methyl salicylate & 0.65 & 0.63 & 0 & 1.15 & 1.53 & 1.78 & 0 & 0.82 \\
\hline 5 & 48.5 & Hexanoic acid, 3-hexenyl ester, (Z)- & 0.29 & 1.91 & 0.24 & 0.4 & 0 & 0.74 & 0 & 0 \\
\hline 6 & 48.89 & Hexanoic acid, hexyl ester & 0 & 0.55 & 0 & 0.13 & 0 & 0 & 0 & 0 \\
\hline 7 & 63.35 & Phthalic acid, diisobutyl ester & 0 & 0 & 0 & 0 & 0 & 0 & 0 & 0 \\
\hline 8 & 64.07 & Hexadecanoic acid, methyl ester & 0 & 0.06 & 0 & 0 & 0.08 & 0.12 & 0 & 0 \\
\hline $\begin{array}{l}\text { Total } \\
\text { Alhyde }\end{array}$ & & & 0.94 & 3.16 & 0.24 & 1.68 & 1.61 & 2.64 & 0 & 0.82 \\
\hline 1 & 3.58 & 2-Hexenal & 0 & 0 & 0 & 0 & 0 & 0 & 0 & 0 \\
\hline 2 & 12.49 & Benzaldehyde & 1.01 & 0.65 & 1.27 & 3.09 & 1.46 & 0.99 & 0.77 & 2.22 \\
\hline 3 & 19.28 & Benzeneacetaldehyde & 0.59 & 0.83 & 0.74 & 0.7 & 0.27 & 0.4 & 0 & 0.37 \\
\hline 4 & 24.04 & Nonanal & 0 & 0 & 0 & 0 & 0 & 0 & 0 & 0 \\
\hline 5 & 34.43 & Decanal & 0.32 & 0.49 & 0 & 0.39 & 0.44 & 0.59 & 0 & 0.21 \\
\hline 6 & 34.73 & 1-p-Menthen-9-al & 0 & 0.12 & 0 & 0.33 & 0.6 & 0 & 0 & 0 \\
\hline Total & & & 1.93 & 2.09 & 2.01 & 4.51 & 2.77 & 1.98 & 0.77 & 2.8 \\
\hline Other & & & & & & & & & & \\
\hline 1 & 2.32 & 1-ethyl-1 $H$-Pyrrole & 1.29 & 0.55 & 1 & 0.72 & 0.56 & 0.33 & 0.56 & 5.82 \\
\hline 2 & 3.98 & $p$-Xylene & 0 & 0 & 0 & 0 & 0 & 0 & 0 & 0 \\
\hline 3 & 19.68 & 1-ethyl- $1 H$-Pyrrole-2-carboxaldehyde & 2.31 & 0.83 & 1.22 & 2.03 & 1.14 & 0.53 & 1.1 & 9.2 \\
\hline 4 & 23.31 & 1-Methyl-4-(1-methylethenyl)-benzene & 0 & 0.79 & 0 & 2.28 & 2.49 & 0 & 0 & 1.91 \\
\hline 5 & 25.53 & 4-Methoxy-N-methyl-benzenamine & 0 & 0 & 0 & 0 & 0 & 0 & 0 & 2.1 \\
\hline 6 & 28.06 & Benzyl nitrile & 1.24 & 2.88 & 2.41 & 9.64 & 1.31 & 0.95 & 1.22 & 0 \\
\hline 7 & 29.55 & Nerol oxide & 0 & 0 & 0 & 0 & 0.55 & 0 & 0 & 0 \\
\hline 8 & 41.42 & Indole & 7.34 & 19.54 & 1.08 & 7.58 & 1.86 & 6.59 & 2.19 & 0 \\
\hline 9 & 55.8 & Dibenzofuran & 0 & 0 & 0 & 0 & 0 & 0 & 0 & 0.22 \\
\hline 10 & 58.36 & Caryophyllene oxide & 0 & 0 & 0 & 0 & 0 & 0 & 0 & 0 \\
\hline 11 & 60.64 & 2,2',5,5'-Tetramethyl-1,1'-biphenyl & 0 & 0 & 0 & 0 & 0 & 0 & 0 & 0 \\
\hline 12 & 63.12 & Caffeine & 0 & 0 & 0 & 0 & 0 & 0 & 0 & 0 \\
\hline total & & & 12.18 & 24.59 & 5.72 & 22.25 & 7.91 & 8.4 & 5.07 & 19.25 \\
\hline Alkane & & & 0 & 0 & 0 & 0 & 0 & 0 & 0 & 0 \\
\hline 1 & 32.87 & Dodecane & 0 & 0 & 0 & 0 & 0 & 0 & 0 & 0 \\
\hline 2 & 41.49 & Tridecane & 0 & 0 & 0 & 0 & 0 & 0 & 0 & 0 \\
\hline 3 & 49.76 & Tetradecane & 0.37 & 0.33 & 0 & 0.43 & 0 & 0 & 0 & 0 \\
\hline 4 & 55.19 & Pentadecane & 0 & 0 & 0 & 0 & 0 & 0 & 0 & 0 \\
\hline 5 & 59.36 & Hexadecane & 0.17 & 0.29 & 0.18 & 0.2 & 0.26 & 0.15 & 0.12 & 0.18 \\
\hline Total & & & 0.54 & 0.62 & 0.18 & 0.63 & 0.26 & 0.15 & 0.12 & 0.18 \\
\hline
\end{tabular}

\subsection{Characteristic Volatile Compounds of the Fresh Leaves and Manufactured Teas with Different Aroma Types}

In order to better visualize the structure and similarities among the samples, hierarchical cluster analysis (HCA) was performed with the full data set. It was clear that the volatile components of fresh leaves had significant differences in relative abundance that were related to aroma type (Figure $4 \mathrm{~A})$.

Volatiles of the fresh leaves included 14 alcohols, six aldehydes, five ketones, five alkanes, 19 alkenes, and six esters. The relative contents of aromatic components in eight types of fresh leaves were very different (Table 2). Linalool, linalool oxide, 2-hexenel, 3-hexeyl acetate, methyl salicylate, geraniol, and nerolidol were the major components, accounting for $81.60-92.00 \%$. Linalool (34.20-42.00\%) was the highest content of fresh leaves, followed by 2 -hexenel (11.42-24.34\%), and methyl salicylate (4.25-20.14\%) (Table 2).

XingRen, HuangZhi, FengHuang Shuixian, and JiangHua were clearly separated in manufactured teas. In the manufactured teas, ten alcohols, four aldehydes, five ketones, five alkanes, 22 alkenes, and four esters were detected. Hotrienol, linalool, D-limonene, linalool oxide, $\beta$-myrcene, $\beta$-ocimene, benzyl nitrile, indole, jasmone, jasmine lactone, and nerolidol were the major components, accounting for
$80.80-87.90 \%$. Hotrienol constituted the highest amount (15.66-72.56\%), followed by linalool (3.67-12.78\%), and D-limonene (3.07-13.93\%) (Table 2).

Contents of the major and characteristic components were very different in eight types of FengHuang Dancong manufactured tea. As shown in Figure 4B, geraniol was the characteristic compound in MiLan. Jasmine lactone, ionene, $\alpha$-farnesene, 2-methyl-6-methylene-1,7-octadien-3-one, indole, nerolidol, hexanoic acid, and 3-hexenyl ester were characteristic components in XingRen. Jasmone, linalool, linalool oxide I, and linalool oxide II were characteristic in GuiHua. Phenylpropanoid/benzenoid derivatives, such as benzaldehyde, phenylethyl alcohol, and benzyl nitrile were the characteristic compounds in HuangZhi. 1- $p$-Menthen-9-al, cosmene, 1,3,8-p-menthatriene, and nerol oxide were the characteristic compounds in YeLai. $\alpha$-Calacorene, copaene, cadinene, hexadecanoic acid, methyl ester, pyranoid linalool oxide, and caryophyllene were the characteristic compounds of YuLan. Hotrienol was the characteristic compound of JiangHua. Most terpenes and pyrrole derivatives were the characteristic compounds in FengHuang Shuixian. Most notably, the aroma profile of FengHuang Shuixian manufactured tea was quite different from the other types. Content of alkenes $(51.97 \%)$ was the highest in FengHuang Shuixian oolong tea; content of alkenes ranged from $8.99-30.66 \%$ in other varieties. The content of alcohol in 
FengHuang Shuixian was $23.82 \%$ but reached $42.28-82.46 \%$ in the other tea types. Notably, the content of 1-ethyl-1H-pyrrole and 1-ethyl-1H-pyrrole-2-carboxaldehyde reached $5.82 \%$ and $9.20 \%$, respectively, in FengHuang Shuixian while the other tea varieties only had $0.33-1.29 \%$ and $0.53-2.31 \%$, respectively, of these compounds (Table 2).

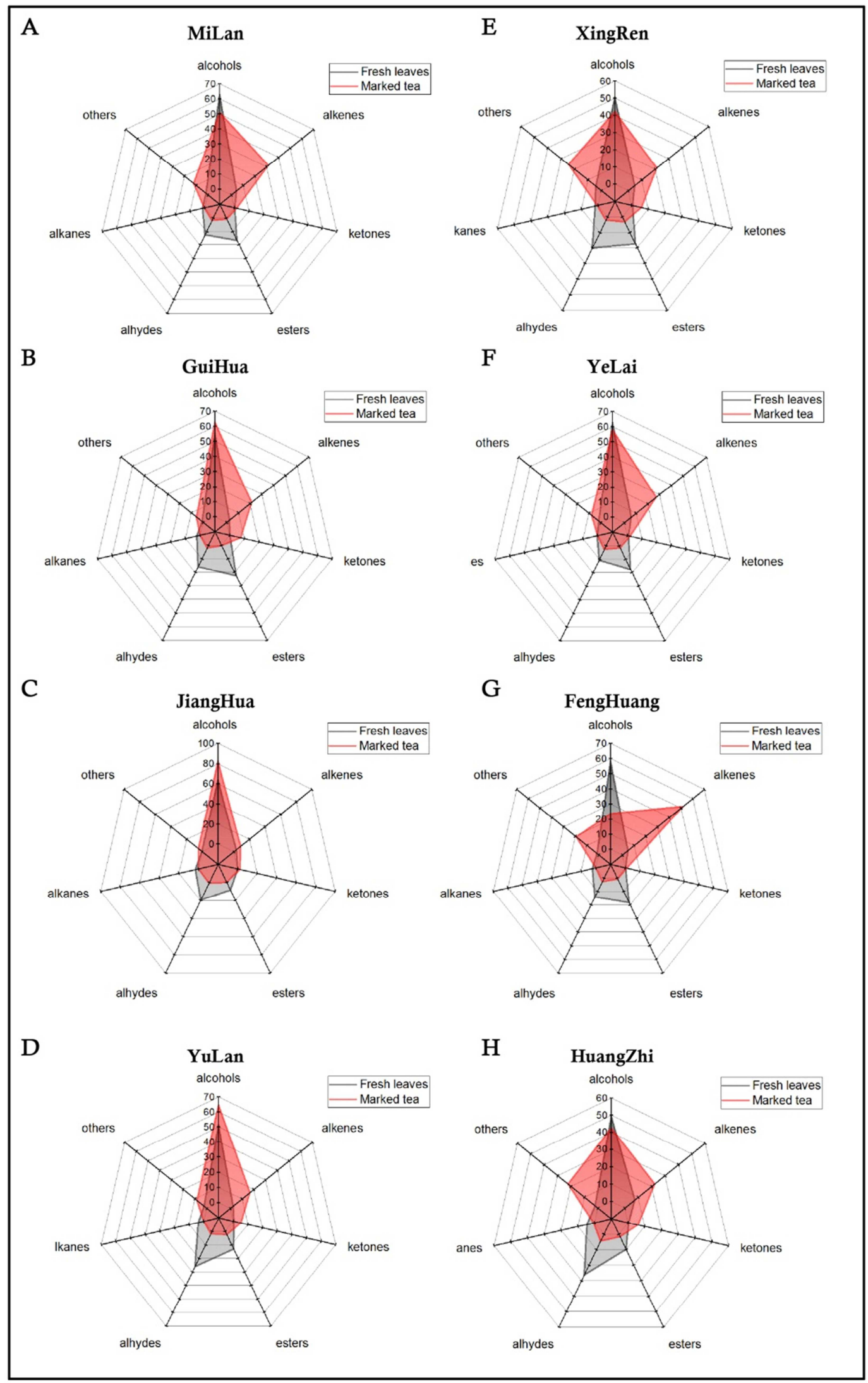

Figure 3. PCA scores plot of fresh leaves (F-) and manufactured tea (M-) of eight types of FengHuang Dancong tea. 
In both the fresh leaves and manufactured teas, GuiHua, MiLan, and HuangZhi samples were not distinctly separated (Figure 2), indicating that their aromatic profiles are very similar. The characteristic compounds of manufactured teas were related to the fresh leaves (Figure $4 \mathrm{~A}$ and $4 \mathrm{~B}$ ), such as geraniol in MiLan, indole and 2-methyl-6-methylene-1,7-octadien-3-one in XingRen, linalool and linalool oxide in GuiHua, and 1-p-menthen-9-al in YeLai. These components are important both in fresh leaves and manufactured teas. Interestingly, linalool and linalool oxide were the characteristic compounds in the fresh leaves of JiangHua but in the manufactured tea, hotrienol, the oxide of linalool, was the characteristic compound.

\section{Discussion}

Similarities and differences of volatile compounds between fresh leaves and manufactured oolong teas were identified in this study. Aroma type may play an important role in these differences. Alcohols were the most important components both in fresh leaves and manufactured teas of eight types of Dancong tea except FengHuang Shuixian manufactured tea (Figure 3). Alcohols are generally characterized by intense sensory descriptions and are associated with citrusy, fatty, and sweet odors [7]. Linalool, which plays an important role both in fresh leaves and manufactured tea, was recognized as one of the key odorants in several studies of black tea leaves and tea infusions, green tea, pu-erh raw tea, and contributes to citrus and floral aroma notes of teas [16]. Hotrienol, the most important compound in the eight types of Dancong manufactured teas but not detected in fresh leaves, may be produced by linalool oxidation during the fermentation procedure. Hotrienolis one of the major compounds in green oolong tea and can be relied on for distinguishing teas obtained from non-fermentation tea processing [17].

Jasmone has been identified as an important fatty acid derivative of the jasmine-like aroma of semifermented teas. Jasmone and jasmine lactone also increased in the manufactured teas, providing a floral aromatic odor [18]. Indole also has a flower-like aroma at a very low concentration $[19,20]$. The content of indole was related to the degree of fermentation in a previous study. Indole increased quickly at the beginning of fermentation in oolong tea and then slowly decreased with continuing fermentation [21].

1-ethyl-1H-pyrrole

and 1-ethyl- $1 H$-pyrrole-2-carboxaldehyde provide burnt and sweet odors [22], which were characteristic components of manufactured teas. The two aromatic compounds were formed by a Maillard reaction with some soluble sugars and amino acids as substrates. The amount of pyrroles increased following increases in time and the temperature of thermal treatment. We found that the pyrroles were at a higher percentage in FengHuang Shuixian manufactured tea (15.02\%) than in the other tea types (0.86-3.60\%). FengHuang Shuixian manufactured tea had the worst aroma, lacking flower aroma and honey taste. This bad aroma maybe associated with the high content of pyrrole derivatives.

Saturated hydrocarbons usually make a minor contribution to the tea aroma, whereas unsaturated hydrocarbons are important contributors to the tea aroma [23].

D-limonene is considered an important volatile and is described as having a "lemon-like" aroma. Moreover, $\beta$-ocimene, another important volatile component, generates a strong "warm herbaceous" aroma [16]. Alkanes and polycyclic aromatic hydrocarbons, such as tridecane, tetradecane, and pentadecane have no effect on tea aroma while terpene compounds possibly play an important role [24] These findings show that terpene compounds may be major contributors to the aroma of oolong tea [25].

Methyl salicylate appears only in teas that have at least a medium degree of fermentation but cannot be detected in unfermented or lightly fermented teas [21]. However, our results showed that methyl salicylate was present in fresh leaves at high concentrations but decreased after processing (Table 2). The results were similar with the research of Huang [26] who found that methyl salicylate was one of the major volatile components in the fresh leaves of different oolong teas using the SDE extraction method. Methyl salicylate was one of the major volatile components in fresh leaves using the HS-SPME method in eight FengHuang Dancong teas. C6 aldehydes, such as 2-hexenal, are considered to be detrimental volatile aroma compounds, and levels of these compounds are found to increase during the processing of oolong tea [27]. Interestingly, we found that C6 aldehydes were not detectable in any manufactured teas.

Production processes affect the aroma formation of oolong tea, which relate to chemical reactions [28]. In the present study, the floral aroma increased, and the grassy odors decreased during the tea manufacturing process. These results were consistent with those reported by Wang et al. [21, 29]. Tea leaf lipids are hydrolyzed, oxidized, and decomposed by a series of enzymatic reactions during the withering process. These reactions produce grassy odors in tea leaves. Linalool and geraniol are liberated from their glucosides by tea leaf glucosidase. Also, other aroma volatiles such as benzylalcohol, 2-phenylethanol, and methylsalicylate may be produced by hydrolysis of non-volatile compounds. These compounds are possibly principal contributors to the sweet floral aroma of oolong teas.

The major components we found were different from other studies on FengHuang Dancong teas [30, 31]. The number of alkenes was significantly higher than in other studies. The differences may be because different extraction and sample preparation methods (HS-SPME or SDE) were used.

Chemometric tools (like stepwise HCA and PCA) showed the separation of FengHuang Dancong teas. This study revealed that differences in aromatic profiles may be obvious for tea varieties with very similar origins. The volatile components of FengHuang Shuixian manufactured tea were obviously different from the other tea varieties, which is consistent with the results of sensory evaluation. We speculate that the higher content of alkene and pyrrole derivatives and lower content of alcohols may cause the bad sensory evaluation of this variety. 


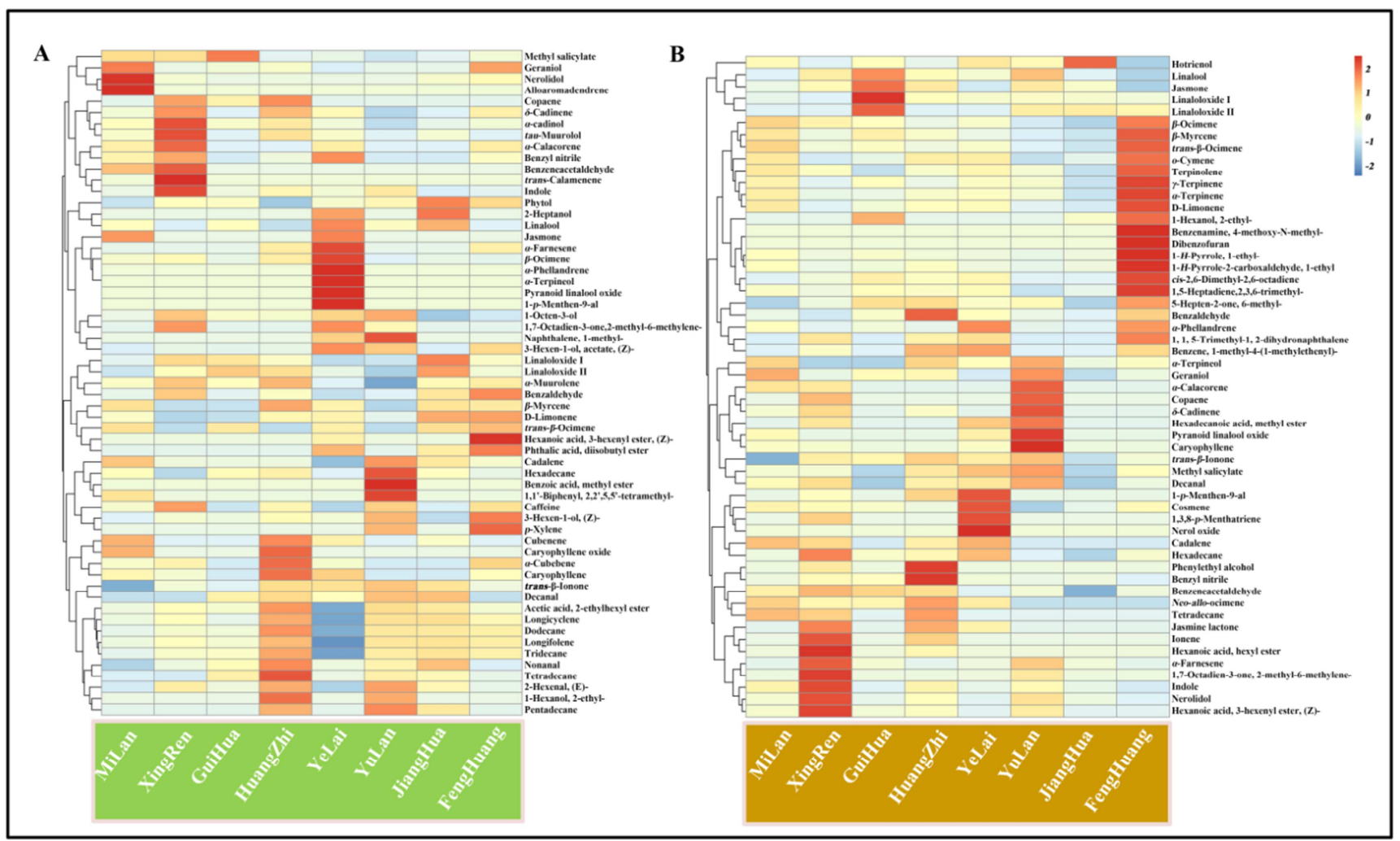

Figure 4. Heatmap and clustering of volatile compounds in eight FengHuang Dancong fresh leaves (A) and manufactured teas (B).

Each row represents a compound and each column represents a type of FengHuang Dancong. Values were centered and scaled in the row direction to form virtual colors as presented in the color key. Compounds with similar contributions were clustered together.

Our results also showed a correlation of the characteristic compounds between manufactured teas and fresh leaves (Figure 4). The correlations indicated that the volatile organic compounds profile of fresh leaves may affect the quality of the manufactured tea and thus require further study to determine if the volatile compounds of fresh leaves can be used as one standard to choose better varieties and processes to make higher quality teas.

\section{Conclusion}

In conclusion, we compared the volatile compound profiles of eight types of FengHuang Dancong tea. Among aroma-active compounds, linalool, (E)-2-hexenal, cis-3-hexenyl acetate, linalool oxide, methyl salicylate, geraniol, and nerolidol were the major volatile components in fresh leaves. But in manufactured teas, hotrienol, linalool, $\beta$-myrcene,

D-limonene, 1-ethyl-1H-pyrrole-2-carboxaldehyde, $\beta$-ocimene, linalool oxide, benzyl nitrile, indole, jasmone, and nerolidol were the major volatile components. HCA analysis showed that relative contents of the major and characteristic components were remarkable different in eight types of FengHuang Dancong fresh leaves and manufactured teas. Most notably, the aroma profile of FengHuang Shuixian manufactured tea was quite different from other aroma varieties, and the difference may be associated with the higher contents of alkene and pyrrole derivatives; lower content of alcohols, especially terpene alcohols in FengHuang Shuixian. Each aroma type had unique aroma characteristics. We also found that the oolong tea processing not only increased the floral aroma but also decreased the grassy odor; in addition, the relative content of methyl salicylate decreased, and alkenes increased after processing. The characteristic compounds between manufactured teas and fresh leaves were closely correlated. But the clear metabolic changes of volatile components between fresh leaves and manufactured teas requires further investigation.

\section{Materials and Methods}

\subsection{Chemicals}

2-Hexenal $(\geq 95 \%), \quad \alpha$-terpinene $(\geq 90 \%), \quad$ D-limonene $(\geq 99 \%), \quad \gamma$-terpinene $(\geq 85 \%)$, benzyl nitrile $(\geq 98 \%)$, cis-jasmone $(\geq 92 \%)$ and caryophyllene ( $\geq 90 \%)$ were purchased from Shanghai Anpel Co., Ltd; $\beta$-ocimene $(\geq 90 \%)$, benzaldehyde ( $\geq 99.5 \%), \beta$-myrcene $(\geq 95 \%), \alpha$-phellandrene ( $\geq 95 \%)$, methyl salicylate $(\geq 99 \%), \sigma$-cymene $(\geq 95 \%)$, linaloloxide $(\geq 97 \%)$, linalool $(\geq 97 \%)$, nerolidol $(\geq 98 \%)$, geraniol $(\geq 98 \%)$ and phenylethyl alcohol (pharmaceutical secondary standard) were purchased from Sigma-Aldrich; cis-3-hexenyl acetate $(100 \mathrm{mg} / \mathrm{L}$ dissolved in $\mathrm{MeOH})$ was purchased from o2si smart solutions; indole ( $\geq 99.9 \%)$ was 
purchased from Supelco; and jasmine lactone ( $\geq 97 \%)$ was purchased from Wako Pure Chemical Industries, Ltd. Ultra-pure water was obtained from a Milli-Q purification system (Millipore, Bedford, USA).

\subsection{Plant Materials and Samplings}

Fresh leaves of eight different types of Dancong tea were collected from reliable sources in FengHuang town of Chao'an county in Guangdong Province. The tea trees, which were between 30-50 years old, were all planted at an elevation of 8,000 feet. One strain of each aroma type was planted in a double line. Fifty trees of one strain were planted in one line. Three replicates of ten out of 100 trees were selected randomly. Fresh leaves from eight types of FengHuang Dancong tea were sampled and kept in liquid nitrogen.

The processing procedure to make oolong tea was as follows: tea leaves were plucked. After the tea leaves were exposed to sunlight for 30 min using a solar withering process, the leaves were turned over and collided with each other six times. Then the leaves were parched at $280{ }^{\circ} \mathrm{C}$ for $30 \mathrm{~min}$ to inactivate enzymatic activity. Leaves were then dried at $120{ }^{\circ} \mathrm{C}$ for $40 \mathrm{~min}$ and further dried at $105{ }^{\circ} \mathrm{C}$ for $1 \mathrm{~h}$ to produce oolong tea products. The manufactured teas were sampled from the local factory, and those teas were processed using the same manufactured techniques. The manufactured teas and fresh leaves were ground to powder using liquid nitrogen and then kept at $-80{ }^{\circ} \mathrm{C}$ until use.

\subsection{Sensory Evaluations}

According to the NY/T 787-2004, the appearance of the tea was evaluated. Three grams of tea sample was extracted with $250 \mathrm{~mL}$ of $100{ }^{\circ} \mathrm{C}$ distilled water for five min. The extracted tea infusion was filtered and cooled to room temperature. Then, five evaluators identified the perceived quality scores of the extracted tea infusions based on the color, taste, and aroma of tea infusions (total score is 100 points). Higher scores mean better quality tea.

\subsection{HS-SPME Analysis}

The fiber coatings $\quad(30-50 \quad \mu \mathrm{m}$ divinyl benzene-carboxyl-polydimethyl siloxane (DVB-CAR-PDMS) were purchased from Supelco (57328U, Supelco). Prior to analyses, the fibers were equilibrated for $60 \mathrm{~min}$ at the manufacturer's recommended conditioning temperature.

Tea powder $(200 \mathrm{mg})$ from each sample was extracted with $1.8 \mathrm{~mL}$ water in the headspace solid-phase microextraction (HS-SPME) device (57357U, Supelco), which included one manual SPME holder (57330U, Supelco), the SPME fiber, and one screw top vial (316018E-2170-10, ANPEL Laboratory Technologies Inc.) with PTFE/silicone septa (606050ULB-18-10, ANPEL Laboratory Technologies Inc.), and a Teflon cover (5310-18-10, ANPEL Laboratory Technologies Inc.). The SPME fiber was exposed to the tea sample headspace while the tea powder kept in the water was continuously heated on the heating block (IKA C-MAG HS 7) for $60 \mathrm{~min}$ at $85{ }^{\circ} \mathrm{C}$ and vigorously stirred. After extraction, the SPME fiber coating was immediately removed from the headspace vial and inserted into the GC injector for preconditioning for five $\min \left(250{ }^{\circ} \mathrm{C}\right)$. The experiment was carried out in triplicate for each tea sample.

\subsection{GC-MS Analysis}

Volatile compounds were analyzed using a 7890A gas chromatograph equipped with a 5975C mass spectrometer (GC-MS, Agilent Technologies, CA, USA). The compounds were separated using an HP-5 MS column $(30 \mathrm{~m} \times 0.25 \mathrm{~mm}$ i.d., film thickness $0.25 \mu \mathrm{m}$; stationary phase: diphenyl-95\% dimethyl siloxane copolymer; Agilent) with helium (percentage purity $>99.999 \%$ ) acting as the gas carrier; the flow rate was controlled at $1 \mathrm{~mL} / \mathrm{min}$. The injection temperature was maintained at $250{ }^{\circ} \mathrm{C}$; the detector temperature was $280{ }^{\circ} \mathrm{C}$; the ion source temperature was $230{ }^{\circ} \mathrm{C}$; and the quadrupole temperature was $150{ }^{\circ} \mathrm{C}$. The following temperature increase program was used for the column: $35^{\circ} \mathrm{C}$ for $2 \mathrm{~min}$; then increased to $40{ }^{\circ} \mathrm{C}$ at a rate of $3{ }^{\circ} \mathrm{C} / \mathrm{min}$ and then held at $40{ }^{\circ} \mathrm{C}$ for $3 \mathrm{~min}$; then increased by $3{ }^{\circ} \mathrm{C} / \mathrm{min}$ to $60^{\circ} \mathrm{C}$ and held at $60^{\circ} \mathrm{C}$ for $3 \mathrm{~min}$; then to $90^{\circ} \mathrm{C}$ at $1.5{ }^{\circ} \mathrm{C} / \mathrm{min}$ and held at $90{ }^{\circ} \mathrm{C}$ for $3 \mathrm{~min}$; then to $125^{\circ} \mathrm{C}$ at $2{ }^{\circ} \mathrm{C} / \mathrm{min}$; then to $150{ }^{\circ} \mathrm{C}$ at $5{ }^{\circ} \mathrm{C} / \mathrm{min}$; and finally to $250{ }^{\circ} \mathrm{C}$ at $15^{\circ} \mathrm{C} / \mathrm{min}$. Splitless mode was used. The MS parameters of ionization energy $70 \mathrm{eV}$ with $3.5 \mathrm{scans} / \mathrm{s}$ and mass range $\mathrm{m} / \mathrm{z}$ 40 - 450 were used.

\subsection{Data Analysis}

The constituents of the volatile compounds were identified by matching the mass spectral data with those stored in the NIST11 spectral library and partly confirmed using standard samples of each volatile compound (1-ethyl-1H-pyrrole, benzyl nitrile, 2-hexenal, benzaldehyde, $\beta$-myrcene, $\alpha$-phellandrene, 3 -hexenyl acetate, $\alpha$-terpinene, $\sigma$-cymene, D-limonene, $\beta$-ocimene, $\gamma$-terpinene, phenylethyl alcohol, benzyl nitrile, methyl salicylate, indole, cis-jasmone, caryophyllene, jasmine lactone, linalool, linaloloxide, linaloloxid II, nerolidol, and geraniol).

The data set was processed using different pattern recognition methods, including principal component analysis (PCA) and hierarchical cluster analysis (HCA). Statistical analysis was performed using SIMCA software (V14, Umetrics AB, Umea, Sweden), and figures were constructed using Origin software (version 8.0, Origin Lab Inc., USA).

\section{Acknowledgements}

This work was supported by the Science and Technology Planning Project of Guangdong Province, China (2013B020305005; 2015A020209076, 2016B030303007), and the Presidential Foundation of the Guangdong Academy of Agricultural Sciences, China (201720). 


\section{Appendix}

Table A1. The characteristic fragment ions of volatile compounds.

\begin{tabular}{|c|c|c|}
\hline Compound & Ion fragment ions of volatile authentic standards & Ion fragment ions of volatile compounds in tea samples \\
\hline 1-ethyl-1H-pyrrole & $41.1,53.1,67.0,80.1,95.1$ & $41.0,53.0,67.0,80.1,95.1$ \\
\hline Benzyl nitrile & $51.1,63.1,77.1,90.1,117.1$ & $51.0,63.0,77.0,90.0,117.1$ \\
\hline 2-Hexenal & $41.1,55.1,69.1,83.0,98.0,147.0$ & $41.1,55.1,69.1,83.1,98.1$ \\
\hline Benzaldehyde & $51.1,77.1,105.1$ & $51.0,77.0,105.1$ \\
\hline$\beta$-Myrcene & $41.1,69.1,77.0,93.1,119.1,136.1$ & $41.1,69.1,77.0,93.1,119.1,138.1$ \\
\hline$\alpha$-Phellandrene & $41.0,51.0,65.0,77.0,93.1,105.1,119.1,134.2$ & $41.0,51.1,65.0,77.0,93.1,105.1,119.1,134.2$ \\
\hline 3-Hexen-1-ol, acetate & $43.1,54.0,67.1,82.1$ & $43.1,54.1,67.1,82.1$ \\
\hline$\alpha$-Terpinene & $41.1,65.0,93.1,121.1,136.1$ & $41.0,65.0,93.1,121.1,136.1$ \\
\hline$\sigma$-Cymene & $41.0,51.0,65.0,77.0,91.1,103.1,119.1,134.1$ & $41.0,51.0,65.0,77.0,91.1,103.1,119.1,134.1$ \\
\hline D-Limonene & $41.1,53.1,68.1,79.1,93.1,107.1,119.1,136.1$ & $41.1,53.1,68.1,79.1,93.1,107.1,119.1,136.1$ \\
\hline$\beta$-Ocimene & $41.1,65.1,79.1,93.1,105.1,121.1,136.1$ & $41.1,65.0,79.0,93.1,105$ 。1, 121.1, 136.1 \\
\hline$\gamma$-Terpinene & $41.1,65.0,77.0,93.1,105.1,121.1,136.1$ & $41.0,65.0,77.0,93.0,105.1,119.1,136.2$ \\
\hline Phenylethyl alcohol & $51.1,65.1,78.1,91.1,104.1,122.1$ & $51.0,65.0,78.1,91.1,104.0,122.1$ \\
\hline Benzyl nitrile & $51.1,63.1,77.1,90.1,117.1$ & $51.0,62.9,77.0,90.0,117.1$ \\
\hline Methyl salicylate & $65.1,92.1,120.1,152.1$ & $65.0,92.0,120.0,152.1$ \\
\hline Indole & $50.1,63.1,90.1,117.1$ & $51.0,63.0,90.0,117.1$ \\
\hline cis-Jasmone & $41.1,55.1,67.1,79.1,91.1,110.1,122.1,135.1,149.1,164.1$ & $41.0,55.0,67.0,79.0,91.1,110.1,122.1,135.1,149.1,164.1$ \\
\hline Caryophyllene & $\begin{array}{l}41.1,55.1,79.1,91.1,105.1,119.1,133.1,147.2,161.1 \\
175.2,189.2,204.2\end{array}$ & $\begin{array}{l}\text { 41.1, 55.1, 79.1, 91.1, 105.1, 120.1, 133.1, 147.2, 161.1, 175.1, } \\
189.2,204.1\end{array}$ \\
\hline Jasmine lactone & $43.0,55.0,71.1,81.1,99.0,117.0,150.2,168.1$ & $43.0,55.0,71.0,81.1,99.0,117.0,150.1,168.1$ \\
\hline Linalool & $41.1,55.1,71.1,93.1,107.1,121.1,136.1$ & $41.1,55.1,71.1,93.1,107.1,121.1,136.1$ \\
\hline Linaloloxide & $43.0,59.1,81.1,94.1,111.1,137.1,155.1$ & $43.0,59.0,81.0,94.0,111.1,137.0,155.1$ \\
\hline Nerolidol & $\begin{array}{l}\text { 41.1, 55.1, 69.1, 93.1, 107.1, 121.1, 136.1, 161.1, 175.2, } \\
189.2,204.2\end{array}$ & 41.1, 55.0, 69.1, 93.1, 107.1, 121.1, 136.1, 161.2, \\
\hline Geraniol & $41.1,55.1,69.1,93.1,107.1,123.1,136.1,206.9$ & $41.0,55.0,69.0,93.1,107.1,123.1,136.0,206.9$ \\
\hline
\end{tabular}

\section{References}

[1] Chen, Y. L., Duan, J., Jiang, Y. M., Shi, J., Peng, L., Xue, S., Kakuda, K. (2011). Production, quality, and biological effects of oolong tea (camellia sinensis). Food Reviews International, 27(1): 1-15.

[2] Chen, Y. S., Tasy, H. R., Yu, T. H. (1998). Studies on the formation of special aroma compounds of Pouchung tea made from different varieties. Developments in Food Science, 40(98): 431-442.

[3] Shi, M. N., Gong, S. Y. (2012). Research progress on tea aroma. Journal of Tea, 38(1): 19-23.

[4] Ma, C., Qu, Y., Zhang, Y., Qiu, B., Wang, Y., Xi, C. (2014). Determination of nerolidol in teas using headspace solid phase microextraction-gas chromatography. Food Chemistry, 152(2): 285-290.

[5] Dai, S., Xie, C., Li, Q., Chen, D., Zheng, R. (1998). Analysis on aromatic constituents of five well-known strains from fenghuang dancong tea cultivar. Journal of Tea Science, 18(1), 39-46

[6] Rawat, R., Gulati, A., Kiranbabu, G., Acharya, R., Kaul, V., Singh, B. (2007). Characterization of volatile components of Kangra orthodox black tea by gas chromatography-mass spectrometry. Food Chemistry, 105 (1): 229-235.

[7] Xu, Y. Q., Wang, C., Li, C. W., Liu, S. H., Zhang, C. X. Li, L. W. Jiang, D. H. (2015). Characterization of aroma-active compounds of pu-erh tea by headspace solid-phase microextraction (HS-SPME) and simultaneous distillation-extraction (SDE) coupled with GC-Olfactometry and GC-MS. Food Analytical Methods, 9(5): 1188-1198.

[8] Xiao, Z., Wang, H., Niu, Y., Liu, Q., Zhu, J., Chen, H., Ma, N. (2017). Characterization of aroma compositions in different Chinese congou black teas using GC-MS and GC-O combined with partial least squares regression. Flavour and Fragrance Journal, 32(4): 265-276.

[9] Gu, X., Zhang, Z., Wan, X, Ning, J., Yao, C., Shao, W. (2009). Simultaneous distillation extraction of some volatile flavor components from pu-erh tea samples-comparison with steam distillation-liquid/liquid extraction and soxhlet extraction. International Journal of Analytical Chemistry, 276713.

[10] Zhu, M., Li, E., He, H. (2008). Determination of volatile chemical constitutes in tea by simultaneous distillation extraction, vacuum hydrodistillation and thermal desorption. Chromatographia, 68 (7-8): 603-610.

[11] Wu, Y., Lv, S., Lian, M., Wang, C., Gao, X., Meng, Q. (2016). Study of characteristic aroma components of baked Wujiatai green tea by HS-SPME/GC-MS combined with principal component analysis. CyTA-Journal of Food, 14(3): 423-432.

[12] Mu, B., Zhu, Y., Lv, H. P., Yan, H., Peng, Q. H., Lin, Z. (2018). The enantiomeric distributions of volatile constituents in different tea cultivars. Food Chemistry, 265: 329-336.

[13] Lv, S. D., Wu, Y. S., Jiang, Y. F., Meng, Q. X. (2014). Comparative analysis of aroma characteristics of oolong tea from different geographical regions. Food Science, 35(2): 146-153. 
[14] Yang, Z., Baldermann, S., Watanabe, N. (2013). Recent studies of the volatile compounds in tea. Food Research International, 53(2): 585-599.

[15] Zheng, X. Q., Li, Q. S., Xiang, L. P., Liang, Y. R. (2016). Recent advances in volatiles of teas. Molecules, 21(3): 338.

[16] Wang, C., Zhang, C., Kong, Y., Peng, X., Li, C., Liu, S., Du, L., Xiao, D., Xu, Y. (2017). A comparative study of volatile components in Dianhong teas from fresh leaves of four tea cultivars by using chromatography-mass spectrometry, multivariate data analysis, and descriptive sensory analysis. Food Research Intermational, 100(1): 267-275.

[17] Pripdeevech, P., Machan. T. (2011). Fingerprint of volatile flavour constituents and antioxidant activities of teas from Thailand. Food Chemistry, 125(2): 797-802.

[18] Katsuno, T., Kasuga, H., Kusano, Y., Yaguchi, Y., Tomomura, M., Cui, J., Yang, Z., Baldermann, S., Nakamura, Y., Ohnishi, T., Mase, N., Watanabe, N. (2014). Characterisation of odorant compounds and their biochemical formation in green tea with a low temperature storage process. Food Chemistry, 148(148C): 388-395.

[19] Xu, X., Yan, M., Zhu, Y. (2005). Influence of fungal fermentation on the development of volatile compounds in the puer tea manufacturing process. Engineering in Life Sciences, 5(4): 382-386.

[20] Zhu, J., Chen, F., Wang, L., Niu, Y., Yu, D., Shu, C., Chen, H., Wang, H., Xiao, Z. (2015). Comparison of aroma-active volatiles in oolong tea infusions using GC-Olfactometry, GC-FPD, and GC-MS. Journal of Agricultural and Food Chemistry, 63(34): 7499-7510.

[21] Wang, B. S., Yu, H. M., Chang, L. W., Yen, W. J., Duh, P. D. (2008). Protective effects of pu-erh tea on LDL oxidation and nitric oxide generation in macrophage cells. LWT-Food Science and Technology, 41(6): 1122-1132.

[22] Xiao, Z., Chen, H., Niu, Y., Wu, M., Chang, S., Zhu, J. (2015). Identify the characteristic aroma components of longjing tea by headspace steam distillation extraction and GC-MS/GC-O. Journal of Zhejiang University, 42(6): 714-720.
[23] Wang, C., Lv, S., Wu, Y., Gao, X., Li, J., Zhang, W., Meng, Q. (2016). Oolong tea made from tea plants from different locations in Yunnan and Fujian, China showed similar aroma but different taste characteristics. Springerplus, 5(1): 576.

[24] Alasalvar, C., Topal, B., Serpen, A., Bahar, B., Pelvan, E., Gokmen, V. (2012). Flavor characteristics of seven grades of black tea produced in Turkey. Journal of Agricultural and Food Chemistry, 60(25): 6323-6332.

[25] Zhu, Y., Shao, C. Y., Lv, H. P., Zhang, Y., Dai, W. D., Guo, L., Tan, J. F., Peng, Q. H., Lin, Z. (2017). Enantiomeric and quantitative analysis of volatile terpenoids in different teas (Camellia sinensis). Journal of Chromatography A, 1490: 177-190.

[26] Huang, D., Qi, D., Shen, C., Deng, Y., Wang, X., Li, Y. (2016). The preliminary study of fresh leaves aroma components of different oolong tea varieties. Chinese Agricultural Science Bulletin, 32(10): 189-199.

[27] Ravichandran, R., Parthiban, R. (2000). Lipid occurrence, distribution and degradation to flavour volatiles during tea processing. Food Chemistry, 68(1): 7-13.

[28] Zeng, L., Zhou, Y., Fu, X., Mei, X., Cheng, S., Gui, J., Dong, F., Tang, J., Ma, S., Yang, Z. (2017). Does oolong tea (Camellia sinensis) made from a combination of leaf and stem smell more aromatic than leaf-only tea? Contribution of the stem to oolong tea aroma. Food Chemistry, 237: 488-498.

[29] Wang, L. F., Lee, J. Y., Chung, J. O., Baik, J. H., So, S., Park, S. K. (2008). Discrimination of teas with different degrees of fermentation by SPME-GC analysis of the characteristic volatile flavour compounds. Food Chemistry, 109(1): 196-206.

[30] Zhou, C., Zhuang, D., Guo, S., Zhu, H., Ma, R.,Wu, Q. (2014). Classification and identification of different aromatics in tea made from different cultivar of Fenghuang Dancong. Journal of Tea Science, 34(6): 609-616.

[31] Zhang, X., Tian, Y. E., Gao, F., Shi, B., Chen, R. (2015). Analysis of volatile compounds of Huangzhi aromatic Camellia sinensis cv. Fenghuangdancong tea. Farm Products Processing, 19. 